\section{$\underset{\substack{\text { hommes } \\ \text { \& migrations }}}{ }$}

\section{Hommes \& migrations}

Revue française de référence sur les dynamiques

migratoires

$1281 \mid 2009$

France-Brésil sous l'angle des migrations et de l'altérité

Natifs de la transnationalisation

\title{
Les professionnels français à São Paulo
}

\section{Claudelir Corrêa Clemente}

\section{(2) OpenEdition}

Journals

Édition électronique

URL : http://journals.openedition.org/hommesmigrations/395

DOI : 10.4000/hommesmigrations.395

ISSN : 2262-3353

Éditeur

Musée national de l'histoire de l'immigration

Édition imprimée

Date de publication : 1 septembre 2009

Pagination : 132-141

ISSN : 1142-852X

Référence électronique

Claudelir Corrêa Clemente, "Les professionnels français à São Paulo », Hommes \& migrations [En ligne], 1281 | 2009, mis en ligne le 29 mai 2013, consulté le 03 mai 2019. URL : http:// journals.openedition.org/hommesmigrations/395; DOI : 10.4000/hommesmigrations.395 


\section{Natifs de la transnationalisation Les professionnels français à São Paulo}

Par Claudelir Corrêa Clemente, docteur en Anthropologie et professeur du Département de Sciences sociales (DECIS) de l'université fédérale d'Uberlândia (UFU)

Les cadres des sociétés internationales, qui circulent à travers les villes du monde, constituent une élite. La vie de ces natifs de la transnationalisation du capital se nourrit de la mobilité. Malgré l'importance du travailleur transnational dans la mondialisation, il est pratiquement invisible dans les statistiques migratoires. Secret et discrétion sont des traits indispensables dans le monde des affaires. Il s'agit de faire la lumière sur le réseau social des expatriés français, à travers des entretiens avec ce type de professionnels et des données ethnographiques recueillies dans la ville de São Paulo. 
Lépoque contemporaine est la scène d'un processus croissant de déplacements humains à travers le monde. Les statistiques sur les migrations internationales estiment à 220 millions $^{(1)}$ le nombre de personnes qui habitent en dehors de leur pays d'origine. Les analyses qualitatives révèlent un phénomène à multiples facettes, laissant voir une variété de groupes et d'individus qui s'essayent à la mobilité. Avec l'essor de la mondialisation économique, matérialisé par la complexité croissante des échanges transnationaux, cet univers de mobilité s'est mu en un ensemble de professionnels qualifiés dans les secteurs de la gestion administrative, la recherche scientifique, la technologie, l'enseignement, la prestation de services spécialisés et dans de multiples compétences nées ou développées dans le sillage de la mondialisation. Ils sont embauchés par des universités, des institutions politiques transnationales (ONU, UNESCO et autres) et, surtout, par des sociétés multinationales. Cet article s'intéresse aux réseaux constitués
Au sein du mouvement d'expansion géographique du capitalisme, on trouve une vie sociale changeante, constituée de relations d'amitié, de mariage, de maternité et paternité vécues par ces professionnels. par ces salariés de sociétés transnationales qui, pour des raisons professionnelles, circulent à travers les villes du monde. En dépit du fait qu'il s'agisse d'un contingent aux multiples nationalités, nous concentrerons nos analyses sur les expériences de professionnels français temporairement "basés" à São Paulo.

En général, et indépendamment de leur nationalité, ils sont connus sur le marché comme des expatriés, et ils restent à l'étranger jusqu'à plusieurs années : leurs vies incarnent une intense mobilité. Afin de comprendre la constitution de leurs réseaux, nous avons choisi d'examiner, premièrement, le contexte qui a donné naissance à ce type de poste dans les sociétés et a contribué à accentuer l'importance de ces professionnels dans le monde des affaires ; et deuxièmement, le pouvoir de l'entreprise transnationale, dont le prestige peut faciliter la libre circulation de ses employés dans le monde.

Cette recherche a représenté un défi en termes méthodologiques, car, en tant qu'anthropologue, j'avais l'habitude d'analyser des communautés et/ou des individus vivant plusieurs années au même endroit. Par leur mobilité, ces professionnels finissent par révéler à l'anthropologie un mode d'être et de vivre qui diffèrent de ceux affichés par les individus autochtones. Au sein du mouvement d'expansion géographique du capitalisme, on trouve une vie sociale changeante, constituée de relations d'amitié, de mariage, de maternité et paternité vécues par ces professionnels. 
Les données concernées proviennent d'un ensemble d'informations recueillies au cours des huit années de recherche auprès de professionnels transnationaux. Il s'agit d'informations collectées lors d'interviews réalisées entre 2001 et 2008, au moyen de contacts directs ou virtuels, auxquelles s'ajoute un travail de terrain dans les villes de São Paulo (Brésil), Paris (France) et Londres (RoyaumeUni), entre 2002 et 2007. Pour cet article, j'ai sélectionné les interviews de professionnels français qui travaillent dans des entreprises transnationales françaises établies dans l'État de São Paulo et les ethnographies réalisées lors des évènements sociaux qui ponctuent la vie des expatriés dans cette métropole brésilienne. Les noms des personnes interviewées ont été modifiés, afin de préserver leur vie privée.

\section{L'émergence d'une main-d'œuvre transnationale}

La circulation de professionnels qualifiés est liée à trois facteurs historiques : la mondialisation, qui a multiplié les échanges culturels, économiques et techniques ; la connaissance spécialisée, qui est devenue l'une des principales sources de production et de reproduction du capitalisme ; et la croissance de la richesse et du pouvoir des sociétés multinationales que l'on a pu constater à la fin du $\mathrm{XX}^{\mathrm{e}}$ siècle.

Les multinationales, notamment américaines, ont joué un rôle essentiel dans la formation de ce cadre dirigeant internatio-

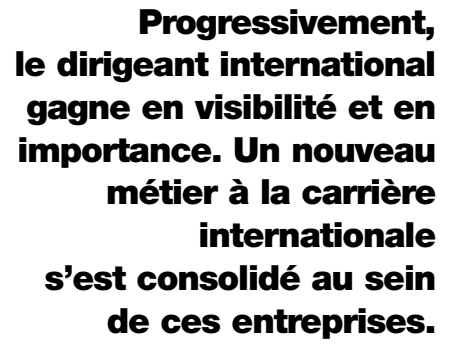
nal. En cultivant certains aspects de la nouvelle culture internationale capitaliste, elles ont impulsé, au niveau de la formation personnelle et professionnelle, un modèle d'exécutif international.

En 1949, la société International Business Machines Corporation (IBM), en réponse à son constant et rapide développement, crée IBM World Trade Corporation, une société totalement indépendante, dont le but est d'augmenter les ventes, services et production au-delà des frontières des États-Unis. Les activités d'IBM World Trade Corporation s'étendent à plus de 150 pays. Cependant, les activités de production, les usines et les laboratoires s'installent dans 15 pays différents.

À partir des années soixante, des dirigeants engagés dans la standardisation de la production et de la consommation participent au développement de ces régions en 
suivant les directives de la société-mère. On assiste à l'émergence d'une maind'ceuvre internationale : un type de dirigeant qualifié pour exercer des fonctions internationales, issu des cadres de l'administration d'IBM.

Durant cette période (allant des années soixante aux années soixante-dix), les grandes décisions stratégiques d'IBM sont prises aux États-Unis - par exemple, celles relatives aux principaux plans d'investissement. De la même façon, les directions régionales et les filiales nationales administrent leurs réseaux commerciaux en fonction des règles dictées par la direction mondiale américaine. Une grande majorité des multinationales européennes adoptent ce mode d'organisation typique des années soixante ${ }^{(2)}$. Progressivement, le dirigeant international gagne en visibilité et en importance. Un nouveau métier à la carrière internationale s'est consolidé au sein de ces entreprises.

\section{Le défi de la mobilité}

Produit des multinationales, ce type de professionnel international, qui aujourd'hui appartient à la vieille garde du monde des affaires, est reconnu comme le grand pionnier, celui qui l'a emporté sur les premières frontières commerciales, missionnaire d'une culture d'entreprise qui a catéchisé un grand nombre d'hommes et de femmes et soumis des nations et des gouvernements à ses doctrines.

Des hommes d'affaires, dont les personnalités affichaient des traits particuliers expression de pouvoir et de glamour - séduisaient leurs contemporains et des nouvelles générations de jeunes dirigeants de différentes catégories. Ils sont devenus synonyme de prestige et ont bénéficié d'une position dominante par rapport aux exécutifs locaux, dont les activités et pouvoirs étaient restreints.

Parmi ces hommes, on trouve Akio Morita (Sony), Jacques Maisonrouge (IBM) et d'autres qui ont fait de leur travail un sacerdoce, un service permanent rendu à la société transnationale. Ils ont fait carrière au sein de leurs entreprises à une époque où la permanence dans une organisation jusqu'à la retraite était perçue comme prestigieuse. La carrière était synonyme de quelque chose qui perdurait toute une vie. On n'avait pas les inquiétudes d'aujourd'hui où le marché devient de plus en plus flexible afin de satisfaire les préférences des consommateurs. Ceux-ci, à leur tour, étant plus conscients de leurs droits.

Héritier de cette génération, le français Roger Granier, président d'une transnationale française du secteur du verre basée à São Paulo, a travaillé pendant presque 35 ans dans le réseau de la même société, dans ses filiales en France. Il se déplaçait à l'intérieur du pays et visait un poste à l'étranger. "Professionnellement, c'est très 
important. On peut rester dans la même ville, au même endroit, dans la même société... Mais bon, ce n'est pas ma conception du travail. Je préfère changer de travail, d'endroit, de ville, je crois que c'est mieux pour ma tête! Dès le début, jai demandé à la société de m'envoyer à l'étranger. Tous les cinq ans, je leur disais : 'J'aimerais bien travailler ailleurs... dans un autre pays'. Et à chaque fois j'entendais : 'Oui, mais vous faites du bon travail en France.' Jusqu'à ce qu'un jour le directeur des ressources humaines m'appelle et me dise : 'J'ai quelque chose pour vous en Amérique latine, au Brésil.' J'avais 53 ans, mais jai accepté le challenge. Prendre ma retraite après avoir eu une expérience de direction internationale, c'est tout ce que jai toujours voulu."

L'obtention d'un poste à l'étranger, en tant que directeur, a symbolisé pour Roger Granier un changement de cap dans sa carrière et de nouveaux bénéfices personnels et professionnels.

Sa mutation a lieu en 2001, alors qu'il a déjà 35 années d'ancienneté dans sa société. 35 ans de travail dans un pays économiquement stable. À l'instar de tous ses contemporains et compatriotes, il avait bénéficié des avantages d'un État de bien-être social et d'un capitalisme développé.

\section{Accumulation du capital et flexibilité du travail}

Pour quelqu'un dans sa situation, qu'est-ce qu'impliquerait un poste international au Brésil, pays connu pour ses inégalités, son manque de sécurité, bref avec toutes les difficultés d'un pays du soi-disant "tiers-monde", encore visibles dans ce nouveau millénaire?

Roger a réfléchi sur l'expérience brésilienne: "En France, si on a un problème, on fait une réunion, une deuxième, des dizaines de réunions, çà et là, et les choses prennent un certain temps pour changer. Ici, les choses sont plus rapides. Les Brésiliens acceptent les changements plus facilement que les Français. C'est vrai qu'ici le type de management est différent. Je trouve que c'est à la fois une qualité et une difficulté. Difficulté parce qu'on doit changer vite. Les Brésiliens changent de direction rapidement et s'adaptent avec la même vitesse. J'ai appris de cela. En France, pour changer quoi que ce soit, il faut organiser une réunion avec le syndicat, discuter avec lui, se réunir avec les gens de l'entreprise, et tout ça pour un problème mineur. Ici, au Brésil, on a plus de souplesse. Ça ne veut pas dire qu'en France on ne soit pas flexible, mais le Français a un problème, il a de la 'certitude ${ }^{(3)}$. On s'attache à nos siècles d'histoire, mais il faut faire attention."

Roger Granier a commencé à travailler à l'époque du fordisme. Paradoxalement, pourtant, dans son récit nous constatons que l'expérience d'un Brésil flexible, aux 
syndicats faibles et aux rapports de travail souples, reflète des changements qui ont eu lieu au sein du régime d'accumulation capitaliste et qui ont touché la vie de professionnels comme lui. En d'autres termes, le travail au Brésil a révélé l'accélération de la flexibilité du travail et de la production.

Depuis longtemps, la logique capitaliste tente de se passer des réunions avec les syndicats. Ce phénomène, perceptible derrière des inquiétudes de notre témoin, atteste de la transition du fordisme vers l'accumulation flexible. Il s'agit de comprendre l'impact de cette dernière sur le profil de ce genre de travailleur - qui, à partir de maintenant, n'est plus international, dans le sens d'être entre les nations, mais transnational, dans le sens où cette personne travaille et agit pour un capital qui se trouve partout.

Dans la mesure où nous parlons de transnationalisation, il convient de citer Canclini : "La transnationalisation est un processus qui se constitue moyennant l'internationalisation de l'économie et de la culture mais qui, à partir de le deuxième moitié du $X X^{e}$, fait quelques pas au-delà, en générant des organismes, des entreprises et des mouvements dont le siège ne se trouve pas exclusivement dans une nation. Philips, Ford ou Peugeot englobent plusieurs pays et agissent avec beaucoup d'indépendance par rapport aux États et aux populations avec lesquels ils s'associent."

\section{Le Brésil dans la carte transnationale}

Dans cette cartographie transnationale, le Brésil figure comme un des pays ouverts à la mondialisation économique. En effet, on a pu constater que la vague de privatisations des sociétés étatiques brésiliennes et l'ouverture du secteur des télécommunications, entamées au milieu des années quatre-vingt-dix, ont attiré vers le pays un segment significatif de la main-d'ceuvre étrangère.

Selon un reportage publié en 2000 par le magazine brésilien d'affaires Exame: "Le Brésil est devenu la destination d'un nouveau flot d'immigrants du monde entier. [...] Actuellement, et d'après les estimations du ministère du Travail et de l'Emploi, on décompte un total de 19000 professionnels étrangers munis d'un visa temporaire en vue d'exercer leur métier dans le pays. Si on les compare avec les immigrants qui se sont installés ici, au cours de plusieurs vagues d'arrivées, dans la période qui va de la fin du XIX à l'après-guerre, il s'agit d'un contingent réduit. Ensemble, ils ne rempliraient que la moitié du Stade de Pacaembu, à Sáo Paulo. Ce qui distingue les étrangers d'aujourd'hui, c'est qu'au lieu de la bêche du paysan comme dans le passé, ils arrivent avec leurs ordinateurs portables et leurs PDA, des titres de spécialisation dans les meilleures universités, une 
expérience professionnelle enrichie par des postes dans plusieurs continents et une vision claire de ce que signifie opérer dans une scène de compétition globale(4)."

L'entrée sélective de ces professionnels dans plusieurs pays indique que la mobilité d'une main-d'ceuvre hautement qualifiée est encouragée comme un aspect important du renforcement d'un marché et d'un type de culture transnationale.

Dans ce processus d'encouragement, les élites nationales ne sont pas dissociées des intérêts transnationaux, mais enchevêtrées, engagées dans des stratégies qui garantissent leur reproduction. Comme le signale Dezalay : "Les stratégies internationales sont des stratégies de distinction pour un petit groupe de privilégiés, auquel s'impose un minimum de discrétion sur ce qui fonde leurs privilèges, afin de pouvoir continuer à pratiquer le double jeu du national et de l'international : investir dans l'international pour renforcer leurs positions dans le champ du pouvoir national et, simultanément, faire valoir leur notoriété nationale pour se faire entendre sur la scène internationale. Pour réussir ce coup double, il doivent cultiver à la fois la proximité et la distance avec leurs concitoyens pour convaincre que non seulement ils partagent les mêmes valeurs, mais aussi qu'ils sont les mieux à même de promouvoir les intérêts nationaux dans la compétition internationale ${ }^{(5)}$."

\section{L'instrumentalisation de la vie sociale}

Dans leur recherche de ce prestige, souscrit par les élites nationales, les professionnels transnationaux manient des manières efficaces pour percer les frontières culturelles et linguistiques, usant de connaissances et de stratégies cosmopolites façonnées dans leur jeunesse, en tant qu'élèves d'écoles de commerce.

Il convient de souligner que la reproduction de cette main-d'cuvre qualifiée n'est pas basée uniquement sur la richesse matérielle ou les bonnes affaires. Le capital économique doit être légitimé et assorti de compétences qui permettent au professionnel de s'adapter à différents contextes culturels, avec des formes de négociation qui varient selon les pays.

La littérature pour professionnels transnationaux préconise que, pour comprendre l'autre culture, l'individu transnational doit y pénétrer. Pour cela, il faut maintenir le contact avec le monde local, des contacts parfois artificiels, tels que déjeuner dans des restaurants dont la clientèle est constituée par des membres des élites locales. Le but est d'acquérir une compétence interculturelle qui soit utile dans le rapport avec quelqu'un d'autre qui, le plus souvent, fait partie d'un groupe choisi de la société locale. 
La génération du français Thomas Lainé, 40 ans, gérant administratif d'une multinationale française basée à São Paulo, connaît bien ces principes de la flexibilité et du court terme. Sa trajectoire professionnelle nous donne des précisions sur cette question. Il raconte : “’̀ 22 ans, jai eu mon premier emploi en dehors de la France. J'ai occupé un poste d'assistant dans une banque française à Tokyo. J'y suis resté un an et demi et jai eu la chance d'être embauché par une banque américaine, Morgan Stanley. Après deux années chez Morgan, jai été recruté par la société pharmaceutique française Aventis, à Paris, où je suis resté quatre ans comme contrô-

\section{La littérature pour professionnels transnationaux préconise que, pour comprendre l'autre culture, l'individu transnational doit y pénétrer.} leur financier. Et puis je suis venu ici."

Avec une vie ponctuée de changements d'emploi, Thomas Lainé a bâti son propre chemin professionnel. Pour lui, il est naturel de changer de société lorsque celle-ci, à un moment donné, ne correspond plus aux intérêts du travailleur. De la même façon, il considère normal que la société ne veuille pas garder un employé qui ne convient plus à ses intérêts.

Par ailleurs, il insiste sur le fait qu'un professionnel comme lui devrait avoir plusieurs expériences internationales, dans différents pays, afin d'acquérir un profil transnational. Il a intégré ces notions dans les salles de cours de son école: "J'ai étudié dans une grande école : l'École d'administration de Paris (ESAM), qui a été très importante dans ma formation. Grâce à l'école, je suis parti pour mon premier stage au Japon, où jai appris le japonais.

\section{Entre visibilité et invisibilité : les réseaux d'une élite}

Le récit de Thomas nous montre que les grandes écoles de commerce, notamment celles de niveau supérieur et réputées, mettent en ceuvre un travail éducatif de formation des professionnels transnationaux qui stimule l'apprentissage des langues, les voyages d'études à l'étranger et les stages dans des sociétés renommées.

Ainsi, les étudiants fréquentent le plus tôt possible le monde de l'entreprise et y effectuent des expériences professionnelles concrètes. Il en ressort une relation de type utilitariste avec l'éducation, pour laquelle les seules connaissances qui ont un sens et une valeur sont celles qui permettent l'efficacité au sein de l'entreprise. Le quotidien de la salle de classe promeut des rencontres avec de futurs associés 
dans les affaires. De cette façon, le carnet d'adresses du temps de la faculté peut devenir la liste de contacts professionnels de demain. Ces établissements éducatifs constituent un genre de réseau international, en collaboration avec les grandes organisations et à l'écoute de l'intérêt du marché. C'est à partir des défis issus de ce dernier que la faculté et l'entreprise proposent des plans d'enseignements et de programmes de capacitation. Une fois diplômés, les professionnels transnationaux sont censés mettre en pratique ces compétences dans leurs relations quotidiennes de travail et interagir avec les catégories sociales ciblées, car leur travail et les produits offerts par l'entreprise qui les emploie ne sont pas destinés à tous. "Le marché des spécialistes internationaux est un marché élitiste, protégé par des barrières discrètes mais efficaces. Pour y accéder, il faut être doté de compétences culturelles ${ }^{(6)}$."

\section{Conclusion}

Nous ne pouvons analyser la formation éducative de ces professionnels de manière isolée. Il convient de la concevoir comme une dimension d'un pouvoir transnational. Il s'agit d'investissements qui assurent la reproduction du capital et, par conséquent, celle des hiérarchies sociales.

La société transnationale est un espace de production et de reproduction de ce pouvoir. La main-d'ceuvre internationale y travaille et en est le produit. Nous observons que la société transnationale, selon sa culture institutionnelle, est censée faire entrer le professionnel dans un moule, tout en s'assurant que ce dernier garde une créativité efficace dans le traitement des intérêts de l'entreprise au sein des différents marchés nationaux.

Ainsi, le national devient un terrain sur lequel les agents internationaux font la promotion de la reproduction du pouvoir transnational. Pour y parvenir, les professionnels transnationaux font l'expérience d'une sociabilité qui cultive une forme de capital social sollicité dans plusieurs situations sociales - les dîners, les cocktails, les vernissages, les sorties, les séances de théâtre sélectionnées -, au cours desquelles on noue des amitiés profitables. En s'appropriant cette pratique, les professionnels transnationaux forment des réseaux de collègues à l'intérieur et en dehors de l'organisation pour laquelle ils travaillent.

Ces relations sont invisibles, puisque voilées par des rapports d'amitié. Elles sont difficilement détectables dans les statistiques de la migration internationale. Protégés par le pouvoir des entreprises qui les emploient, les professionnels transnationaux entrent et sortent des pays avec discrétion et efficacité, sans provoquer le moindre remous dans les politiques migratoires. 
Bibliographie

- Almeida Ana Maria F. et Nogueira Maria A. (org.), A escolarizaçâoo das elites. Petrópolis, Vozes, 2002.

- Arjur Appadurai, Après le colonialisme, Paris, Payot, 2001.

- Teresinha Bernardo et Claudelir C. Clemente, Diasporas, redes e guetos:conceitos e configuraçóes no contexto transnacional. São Paulo, EDUC/CAPES, 2008.

- Clemente Claudelir C., "Entre visibilidade e invisibilidade: as redes de profissionais transnacionais", Revista Interdisciplinar de mobilidade humana/REMHU, Ano XVII, n 32, Brasília, 2009.

- Dezalay Yves, "Les courtiers de l'international : héritiers cosmopolites, mercenaires de l'impérialisme et missionaires de l'universel", Sociologie de la mondialisation, actes de la recherche, mars 2004, p. 151-2.

- Tarrius Alain, Les fourmis d'Europe, Paris, L'Harmattan, 1992

- Tarrius Alain, Les nouveaux cosmopolitismes. Mobilités, identités, territoires, Éditions de l'Aube, La Tour d'Aigues, 2000.

- Wagner Anne-Catherine, Les nouvelles élites de la mondialisation, Paris, PUF, 1998.

\section{Notes}

1. Clovis Rossi, "Forum propōe cidadania global do migrante", Folha de São Paulo, Caderno Mundo, 24 août 2008, p. 22.

2. Sur ce sujet, voir aussi Anne-Catherine Wagner, Les nouvelles élites de la mondialisation, Paris, PUF, 1998.

3. En français dans le texte [NDT].

4. "Os novos migrantes", www.exame.com.br, consulté le $1^{\text {er }}$ septembre 2000.

5. Yves Dezalay, "Les courtiers de l'international : héritiers cosmopolites, mercenaires de l'impérialisme et missionaires de l'universel", Sociologie de la mondialisation. actes de la recherche, Actes 151-2, mars 2004, p. 11.

6. Ana Maria F. Almeida et Maria A. Nogueira, (org.), A escolarizaçấo das elites. Petrópolis, Vozes, 2002, p. 29. 